\title{
COMPUTING $\pi(x)$ : THE MEISSEL, LEHMER, LAGARIAS, MILLER, ODLYZKO METHOD
}

\author{
M. DELEGLISE AND J. RIVAT
}

\begin{abstract}
Let $\pi(x)$ denote the number of primes $\leq x$. Our aim in this paper is to present some refinements of a combinatorial method for computing single values of $\pi(x)$, initiated by the German astronomer Meissel in 1870, extended and simplified by Lehmer in 1959, and improved in 1985 by Lagarias, Miller and Odlyzko. We show that it is possible to compute $\pi(x)$ in $O\left(\frac{x^{2 / 3}}{\log ^{2} x}\right)$ time and $O\left(x^{1 / 3} \log ^{3} x \log \log x\right)$ space. The algorithm has been implemented and used to compute $\pi\left(10^{18}\right)$.
\end{abstract}

\section{INTRODUCTION}

One of the oldest problems in mathematics is to compute $\pi(x)$, the exact number of primes $\leq x$. The most obvious method for computing $\pi(x)$ is to find and count all primes $p \leq x$, for instance by the sieve of Eratosthenes. According to the Prime Number Theorem

$$
\pi(x) \sim \frac{x}{\log x}, \quad x \rightarrow \infty .
$$

Therefore, such a method cannot compute $\pi(x)$ with less than about $\frac{x}{\log x}$ operations.

Despite its time complexity, the sieve of Eratosthenes has been for a very long time the practical way to compute $\pi(x)$. In the second half of the 19th century, the astronomer Meissel discovered a practicable combinatorial method that is faster than finding all primes $\leq x$. He used his algorithm to compute by hand $\pi\left(10^{8}\right)$ and $\pi\left(10^{9}\right)$ (which turned out to be too small by 56 ) $[4,5,6,7]$.

In 1959, Lehmer extended and simplified Meissel's method. He used an IBM 701 computer to obtain the value of $\pi\left(10^{10}\right)$ (his value was shown [1] to be too large by $1)$.

In 1985, Lagarias, Miller and Odlyzko [2] adapted the Meissel-Lehmer method and proved that it is possible to compute $\pi(x)$ with $O\left(\frac{x^{2 / 3}}{\log x}\right)$ operations using $O\left(x^{1 / 3} \log ^{2} x \log \log x\right)$ space. They used their algorithm to compute several values of $\pi(x)$ up to $x=4 \cdot 10^{16}$. They also corrected the value of $\pi\left(10^{13}\right)$ given in [1], which was too small by 941 .

In 1987, Lagarias and Odlyzko [3] described a completely different method, based on numerical integration of certain integral transforms of the Riemann $\zeta$-function, for computing $\pi(x)$, using $O\left(x^{1 / 2+\varepsilon}\right)$ time and $O\left(x^{1 / 4+\varepsilon}\right)$ space for each $\varepsilon>0$.

Received by the editor January 12, 1994 and, in revised form, December 1, 1994.

1991 Mathematics Subject Classification. Primary 11N05, 11Y70.

(C)1996 American Mathematical Society 
Despite its asymptotic superiority, this algorithm has never been implemented. Its authors noted [2] that the implied constants are probably large, and therefore that it would not be competitive with their version of the Meissel-Lehmer method for $x \leq 10^{17}$.

In this paper we describe a modified form of the algorithm presented in [2] which computes $\pi(x)$ using $O\left(\frac{x^{2 / 3}}{\log ^{2} x}\right)$ time and $O\left(x^{1 / 3} \log ^{3} x \log \log x\right)$ space.

\section{OutLine of The Method}

For clarity we will describe the whole method given in [2], in order to introduce the quantities needed for the analysis. For the convenience of the reader we adopt the notations used in [2], and follow as long as possible the same approach. In particular, $\S \S 3,4,5$ are close to [2].

The idea that many special leaves (see below, $\S 6$ ) could be computed at the same time, saving much computation (and a $\log x$ factor in the complexity) was also present in [2]. We develop this idea further, and show that it is possible to compute more special leaves at the same time, saving another $\log x$ factor in the complexity (see $\S 6.2$ and below).

\section{The Meissel-Lehmer Method}

Let $p_{1}, p_{2}, p_{3}, \ldots$ denote the primes $2,3,5, \ldots$ numbered in increasing order. Let $\phi(x, a)$ denote the partial sieve function, which counts numbers $\leq x$ with all prime factors greater than $p_{a}$ :

$$
\phi(x, a)=\#\left\{n \leq x ; p \mid n \Rightarrow p>p_{a}\right\}
$$

and let

$$
P_{k}(x, a)=\#\left\{n \leq x ; n=q_{1} q_{2} \cdots q_{k}, q_{1}, \ldots, q_{k}>p_{a}\right\},
$$

which counts numbers $\leq x$ with exactly $k$ prime factors, all larger than $p_{a}$. We set $P_{0}(x, a)=1$.

If we sort the numbers $\leq x$ by the number of their prime factors greater than $p_{a}$, we obtain the following identity:

$$
\phi(x, a)=P_{0}(x, a)+P_{1}(x, a)+\cdots+P_{k}(x, a)+\cdots,
$$

where the sum on the right has only finitely many nonzero terms, because $P_{k}(x, a)=$ 0 for $p_{a}^{k}>x$.

Let $y$ denote an integer such that $x^{1 / 3} \leq y \leq x^{1 / 2}$, and let $a=\pi(y)$.

From $P_{1}(x, a)=\pi(x)-a$ and $P_{k}(x, a)=0$ for $k \geq 3$ we deduce

$$
\pi(x)=\phi(x, a)+a-1-P_{2}(x, a) .
$$

Hence, for computing $\pi(x)$ it remains to compute $\phi(x, a)$ and $P_{2}(x, a)$.

\section{Computing $P_{2}(x, a)$}

By (3) we have to count all pairs $(p, q)$ of prime numbers such that $y<p \leq q$ and $p q \leq x$.

We first remark that $p \in[y+1, \sqrt{x}]$. Furthermore, for each $p$, we have $q \in$ $[p, x / p]$. Thus,

$$
P_{2}(x, a)=\sum_{y<p \leq \sqrt{x}}\left(\pi\left(\frac{x}{p}\right)-\pi(p)+1\right)
$$


When $p \in[y+1, \sqrt{x}]$, we have $\frac{x}{p} \in\left[1, \frac{x}{y}\right]$. Hence, $P_{2}(x, a)$ can be computed by completely sieving the interval $\left[1, \frac{x}{y}\right]$ and then adding up $\pi\left(\frac{x}{p}\right)-\pi(p)+1$ for all primes $p \in[y+1, \sqrt{x}]$. In order to reduce the space complexity of the above method, we can work with blocks of length $L$. For $L=y$, we can compute $P_{2}(x, a)$ in $O\left(\frac{x}{y} \log \log x\right)$ time and $O(y)$ space.

\section{The Sieve MACHINERY FOR COMPUting $\phi(x, a)$}

For $b \leq a$ the set of all integers $\leq x$ whose prime factors are $>p_{b-1}$ is composed of two classes:

1. those that are multiples of $p_{b}$,

2. those not divisible by $p_{b}$.

The first class has $\phi\left(\frac{x}{p_{b}}, b-1\right)$ elements, while the second has $\phi(x, b)$ elements. Hence we conclude:

Lemma 5.1. The function $\phi$ satisfies the following identities:

$$
\begin{aligned}
& \phi(u, 0)=[u], \\
& \phi(x, b)=\phi(x, b-1)-\phi\left(\frac{x}{p_{b}}, b-1\right) .
\end{aligned}
$$

A straightforward method for computing $\phi(x, a)$ can be deduced from this lemma: it suffices to apply repeatedly the recurrence (7) until we get terms of the form $\phi(u, 0)$, which are easy to compute using (6). One may think of this process as creating a rooted binary tree starting with the root node $\phi(x, a)$; see Fig. 1 . Using this method, we obtain the following formula:

$$
\phi(x, a)=\sum_{\substack{1 \leq n \leq x \\ P^{+}(n) \leq y}} \mu(n)\left[\frac{x}{n}\right],
$$

where $\mu(n)$ denotes the Möbius function and $P^{+}(n)$ denotes the greatest prime factor of $n$.

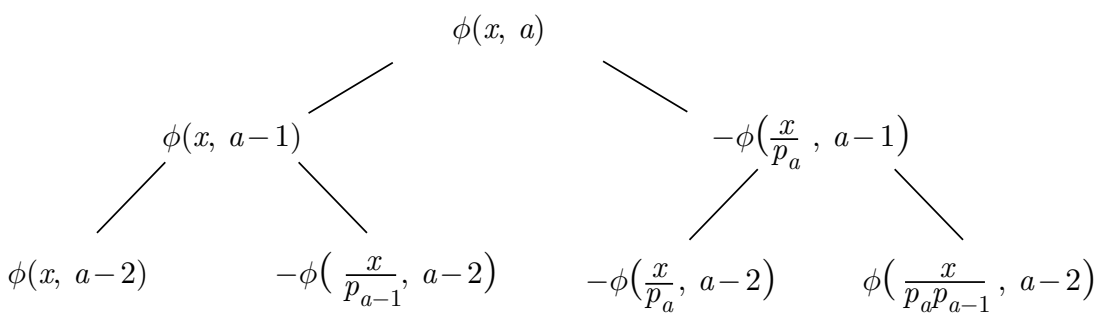

Figure 1. A binary tree for computing $\phi(x, a)$ : the sum of the terminal nodes is $\phi(x, a)$ 
Unfortunately, this sum has too many terms for our purpose: as $y \geq x^{1 / 3}$, if we only count the $n$ 's which are the product of three primes $\leq y$, we get at least about $\frac{x}{\log ^{3} x}$ such terms.

In order to limit the growth of the tree, we must replace the trivial truncation rule,

Truncation Rule 1. Do not split a node $\mu(n) \phi\left(\frac{x}{n}, b\right)$ if $b=0$

with the more powerful:

Truncation Rule 2. Do not split a node $\mu(n) \phi\left(\frac{x}{n}, b\right)$ if either of the following holds:

1. $b=0$ and $n \leq y$,

2. $n>y$.

We are now able to define two clases of leaves:

1. ordinary leaves are those of the form $\mu(n) \phi\left(\frac{x}{n}, 0\right)$ satisfying $n \leq y$,

2. special leaves are those of the form $\mu(n) \phi\left(\frac{x}{n}, b-1\right)$ satisfying $n>y$ with $n=m p_{b}$ and $m \leq y$.

We conclude that:

\section{Lemma 5.2. We have}

$$
\phi(x, a)=S_{0}+S
$$

where $S_{0}$ is the contribution of the ordinary leaves,

$$
S_{0}=\sum_{n \leq y} \mu(n)\left[\frac{x}{n}\right],
$$

and $S$ is the contribution of the special leaves,

$$
S=\sum_{\frac{n}{\delta(n)} \leq y<n} \mu(n) \phi\left(\frac{x}{n}, \pi(\delta(n))-1\right),
$$

where $\delta(n)$ denotes the smallest prime factor of $n$.

The computation of $S_{0}$ can be achieved in $O(y \log \log x)$ time, which is negligible. It remains to compute $S$.

\section{Computing $S$}

We have

$$
S=-\sum_{p \leq y} \sum_{\substack{\delta(m)>p \\ m \leq y<m p}} \mu(m) \phi\left(\frac{x}{m p}, \pi(p)-1\right) .
$$


We now write

$$
S=S_{1}+S_{2}+S_{3}
$$

with

$$
\begin{aligned}
S_{1} & =-\sum_{x^{\frac{1}{3}<p \leq y}} \sum_{\substack{\delta(m)>p \\
m \leq y<m p}} \mu(m) \phi\left(\frac{x}{m p}, \pi(p)-1\right), \\
S_{2} & =-\sum_{x^{\frac{1}{4}}<p \leq x} \sum_{\substack { \frac{1}{3} \\
\begin{subarray}{c}{\delta(m)>p \\
m \leq y<m p{ \frac { 1 } { 3 } \\
\begin{subarray} { c } { \delta ( m ) > p \\
m \leq y < m p } }\end{subarray}} \mu(m) \phi\left(\frac{x}{m p}, \pi(p)-1\right), \\
S_{3} & =-\sum_{p \leq x^{\frac{1}{4}}} \sum_{\substack{\delta(m)>p \\
m \leq y<m p}} \mu(m) \phi\left(\frac{x}{m p}, \pi(p)-1\right) .
\end{aligned}
$$

First observe that the $m$ 's involved in $S_{1}$ and $S_{2}$ are all prime: otherwise, since $\delta(m)>p>x^{1 / 4}$, we would have $m>p^{2}>\sqrt{x}$, a contradiction with $m \leq y$. Moreover, the condition $y \leq p m$ is true when $m p>x^{1 / 2} \geq y$. Hence we have

$$
\begin{aligned}
S_{1} & =\sum_{x^{\frac{1}{3}<p \leq y}} \sum_{p<q \leq y} \phi\left(\frac{x}{p q}, \pi(p)-1\right), \\
S_{2} & =\sum_{x^{\frac{1}{4}}<p \leq x^{\frac{1}{3}}} \sum_{p<q \leq y} \phi\left(\frac{x}{p q}, \pi(p)-1\right) .
\end{aligned}
$$

\subsection{Computing $S_{1}$. Since}

$$
\frac{x}{p q}<x^{1 / 3}<p
$$

we have

$$
\phi\left(\frac{x}{p q}, \pi(p)-1\right)=1 .
$$

Hence all terms involved in $S_{1}$ are equal to 1 . So we have to count all pairs $(p, q)$ such that

$$
x^{1 / 3}<p<q \leq y
$$

Thus,

$$
S_{1}=\frac{\left(\pi(y)-\pi\left(x^{1 / 3}\right)\right)\left(\pi(y)-\pi\left(x^{1 / 3}\right)-1\right)}{2} .
$$

This takes constant time to compute $S_{1}$.

6.2. Computing $S_{2}$. We have

$$
S_{2}=\sum_{x^{1 / 4}<p \leq x^{1 / 3}} \sum_{p<q \leq y} \phi\left(\frac{x}{p q}, \pi(p)-1\right) .
$$


We split $S_{2}$ in two parts, depending on $q>x / p^{2}$ or $q \leq x / p^{2}$ :

$$
S_{2}=U+V
$$

with

$$
U=\sum_{x^{1 / 4}<p \leq x^{1 / 3}} \sum_{\substack{p<q \leq y \\ q>\frac{x}{p^{2}}}} \phi\left(\frac{x}{p q}, \pi(p)-1\right)
$$

and

$$
V=\sum_{x^{1 / 4}<p \leq x^{1 / 3}} \sum_{\substack{p<q \leq y \\ q \leq \frac{x}{p^{2}}}} \phi\left(\frac{x}{p q}, \pi(p)-1\right) .
$$

6.3. Computing $U$. The condition $q>x / p^{2}$ implies $p^{2}>x / q \geq x / y$ and $p>$ $\sqrt{x / y}$. Thus,

$$
U=\sum_{\sqrt{\frac{x}{y}<p \leq x^{1 / 3}}} \sum_{\substack{p<q \leq y \\ q>\frac{x}{p^{2}}}} \phi\left(\frac{x}{p q}, \pi(p)-1\right) .
$$

From $x / p^{2}<q$ we deduce $x / p q<p$ and $\phi(x / p q, \pi(p)-1)=1$. Each term in the sum $U$ equals 1 . Hence,

$$
U=\sum_{\sqrt{\frac{x}{y}}<p \leq x^{1 / 3}} \#\left\{q ; \frac{x}{p^{2}}<q \leq y\right\} .
$$

Thus,

$$
U=\sum_{\sqrt{\frac{x}{y}}<p \leq x^{1 / 3}}\left(\pi(y)-\pi\left(\frac{x}{p^{2}}\right)\right) .
$$

Since $x / p^{2}<y$, the sum $U$ can be calculated in $O(y)$ operations once we have tabulated $\pi(t)$ for $t \leq y$.

6.4. Computing $V$. For each term involved in $V$ we have $p \leq \frac{x}{p q}<x^{1 / 2}<p^{2}$. Hence,

$$
\begin{aligned}
\phi\left(\frac{x}{p q}, \pi(p)-1\right) & =1+\pi\left(\frac{x}{p q}\right)-(\pi(p)-1) \\
& =2-\pi(p)+\pi\left(\frac{x}{p q}\right) .
\end{aligned}
$$

Thus,

$$
V=V_{1}+V_{2}
$$

with 


$$
\begin{aligned}
V_{1} & =\sum_{x^{1 / 4}<p \leq x^{1 / 3}} \sum_{p<q \leq \min \left(\frac{x}{p^{2}}, y\right)}(2-\pi(p)), \\
V_{2} & =\sum_{x^{1 / 4}<p \leq x^{1 / 3}} \sum_{p<q \leq \min \left(\frac{x}{p^{2}}, y\right)} \pi\left(\frac{x}{p q}\right) .
\end{aligned}
$$

Computing $V_{1}$ can be achieved in $O\left(x^{1 / 3}\right)$ time once we have tabulated $\pi(t)$ for $t \leq y$.

In order to speed up the computation of $V_{2}$, we observe that for each $p$ we can split the summation over $q$ into sums over $q$ on intervals where the function $q \mapsto \pi\left(\frac{x}{p q}\right)$ is constant. Thus, we only need the length of these intervals, and the set of values of $q$ where $q \mapsto \pi\left(\frac{x}{p q}\right)$ is changing.

More precisely, we first split $V_{2}$ in two parts in order to simplify the condition $q \leq \min \left(x / p^{2}, y\right)$ :

$$
V_{2}=\sum_{x^{1 / 4}<p \leq \sqrt{\frac{x}{y}}} \sum_{p<q \leq y} \pi\left(\frac{x}{p q}\right)+\sum_{\sqrt{\frac{x}{y}}<p \leq x^{1 / 3}} \sum_{p<q \leq \frac{x}{p^{2}}} \pi\left(\frac{x}{p q}\right) .
$$

We now write

$$
V_{2}=W_{1}+W_{2}+W_{3}+W_{4}+W_{5}
$$

with

$$
\begin{aligned}
W_{1} & =\sum_{x^{1 / 4}<p \leq \frac{x}{y^{2}}} \sum_{p<q \leq y} \pi\left(\frac{x}{p q}\right), \\
W_{2}= & \sum_{\frac{x}{y^{2}}<p \leq \sqrt{\frac{x}{y}}} \sum_{p<q \leq \sqrt{\frac{x}{p}}} \pi\left(\frac{x}{p q}\right), \\
W_{3}= & \sum_{\frac{x}{y^{2}}<p \leq \sqrt{\frac{x}{y}}} \sum_{\sqrt{\frac{x}{p}<q \leq y}} \pi\left(\frac{x}{p q}\right), \\
W_{4}= & \sum_{\sqrt{\frac{x}{y}}<p \leq x^{1 / 3}} \sum_{p<q \leq \sqrt{\frac{x}{p}}} \pi\left(\frac{x}{p q}\right), \\
W_{5}= & \sum_{\sqrt{\frac{x}{y}}<p \leq x^{1 / 3}} \sum_{\sqrt{\frac{x}{p}<q \leq \frac{x}{p^{2}}}} \pi\left(\frac{x}{p q}\right) .
\end{aligned}
$$

Computing $W_{1}$ and $W_{2}$. These two quantities need values of $\pi(x / p q)$ with $y<$ $x / p q<x^{1 / 2}$. They are computed simultaneously with a sieve of the interval $[1, \sqrt{x}]$. The sieving is done by blocks, and for each block we sum $\pi(x / p q)$ for the pairs $(p, q)$ subjected to the conditions of the sums $W_{1}$ or $W_{2}$ and such that $x / p q$ lies in the block.

Computing $W_{3}$. For each $p$ we speed up the computation of the sum over $q$ by computing in $O(1)$ operations the sums of the $\pi(x / p q)$ for the values of $q$ for which $\pi(x / p q)$ is constant. When we obtain a new value of $q$, we compute $\pi(x / p q)$ with 
the table of values of $\pi(t)$ for $t \leq y$. Then a table of all primes $\leq y$ gives $t$ such that $\pi(t)<\pi(t+1)=\pi\left(\frac{x}{p q}\right)$. We then deduce the next value of $q$ for which $\pi(x / p q)$ is changed.

Computing $W_{4}$. We simply sum over $(p, q)$. There would be no advantage to proceed as for $W_{3}$ since most of the values $\pi(x / p q)$ are distinct.

Computing $W_{5}$. We proceed as for $W_{3}$.

\section{Computing $S_{3}$}

We sieve the interval $\left[1, \frac{x}{y}\right]$ successively by all primes less than $x^{1 / 4}$. As soon as we have sieved by $p_{k-1}$, we sum all $-\mu(m) \phi\left(\frac{x}{m p_{k}}, k-1\right)$ for all squarefree $m \in[y / p, y]$ such that $\delta(m)>p_{k}$. This computation can be done by blocks, see [2]. The main idea is that we maintain a binary tree (as explained in $[2$, pp. 545,546$]$ ) in connection with the interval we are sieving, to keep track of the intermediate results after sieving by all primes up to a given prime. It is then possible to know the number of unsieved elements in the interval less than a given value, using only $O(\log x)$ operations.

\section{Time AND SPACE COMPleXity}

The time and space significant computations are:

1. The computation of $P_{2}(x, a)$,

2. The computation of $W_{1}, W_{2}, W_{3}, W_{4}, W_{5}$,

3. The computation of $S_{3}$.

8.1. Cost of computing $P_{2}(x, y)$. We have already seen that it $\operatorname{costs} O\left(\frac{x}{y} \log \log x\right)$ time and $O(y)$ space.

8.2. Cost of computing $W_{1}, W_{2}, W_{3}, W_{4}, W_{5}$. For $W_{1}$ and $W_{2}$ the sieve costs $O(\sqrt{x} \log \log x)$ time and $O(y)$ space, working by blocks of length $y$.

The time necessary to compute the sum $W_{1}$ is about

$$
\pi\left(\frac{x}{y^{2}}\right) \pi(y)=O\left(\frac{x}{y \log ^{2} x}\right) .
$$

The time necessary to compute the sum $W_{2}$ is about

$$
O\left(\sum_{\frac{x}{y^{2}}<p \leq \sqrt{\frac{x}{y}}} \pi\left(\sqrt{\frac{x}{p}}\right)\right)=O\left(\frac{x^{3 / 4}}{y^{1 / 4} \log ^{2} x}\right) .
$$

In $W_{3}$, for each $p$ we have $\frac{x}{p q} \leq \sqrt{x / p}$. Hence, $\pi(x / p q)$ takes at most $\pi(\sqrt{x / p})$ values. For each such value it costs constant time to determine the number of $q$ 's concerned. Hence, the time necessary to compute the sum $W_{3}$ is about

$$
O\left(\sum_{\frac{x}{y^{2}}<p \leq \sqrt{\frac{x}{y}}} \pi\left(\sqrt{\frac{x}{p}}\right)\right)=O\left(\frac{x^{3 / 4}}{y^{1 / 4} \log ^{2} x}\right) .
$$


The time necessary to compute the sum $W_{4}$ is about

$$
O\left(\sum_{\sqrt{\frac{x}{y}}<p \leq x^{1 / 3}} \pi\left(\sqrt{\frac{x}{p}}\right)\right)=O\left(\frac{x^{2 / 3}}{\log ^{2} x}\right) .
$$

We proceed for $W_{5}$ as for $W_{3}$, and the time necessary to compute the sum $W_{5}$ is about

$$
O\left(\sum_{\sqrt{\frac{x}{y}}<p \leq x^{1 / 3}} \pi\left(\sqrt{\frac{x}{y}}\right)\right)=O\left(\frac{x^{2 / 3}}{\log ^{2} x}\right) .
$$

8.3. Cost of computing $S_{3}$. The sieve. Owing to the necessity of quickly retrieving the values $\phi(u, b)$, we have to maintain a data structure such that each access costs $O(\log x)$ instead of $O(1)$ in a normal sieve. Hence the cost is $O\left(\frac{x}{y} \log x \log \log x\right)$.

The sum. For each term in the sum we have to access the data structure mentioned above, doing $O(\log x)$ operations. It remains to count the terms in the sum. All leaves are of the form $\pm \phi\left(x / m p_{b}, b-1\right)$ with $m \leq y$ and $b<\pi\left(x^{1 / 4}\right)$. Hence, the number of these leaves is $O\left(y \pi\left(x^{1 / 4}\right)\right)$.

The total cost of $S_{3}$ is

$$
O\left(\frac{x}{y} \log x \log \log x+y x^{1 / 4}\right)
$$

8.4. Total cost. We have described an algorithm taking $O(y)$ space and

$$
O\left(\frac{x}{y} \log \log x+\frac{x}{y} \log x \log \log x+x^{1 / 4} y+\frac{x^{2 / 3}}{\log ^{2} x}\right)
$$

time.

If we choose $y=x^{1 / 3} \log ^{3} x \log \log x$, the time complexity is $O\left(\frac{x^{2 / 3}}{\log ^{2} x}\right)$.

\section{Practical considerations}

We describe here some modifications which improve the time of computation without changing the asymptotic complexity.

- In the truncation rule 2 , we may replace $y$ by some $z>y$. It is possible to prove that the time complexity for computing $S_{3}$ then becomes

$$
O\left(\frac{x}{z} \log x \log \log x+\frac{y x^{1 / 4}}{\log x}+z^{3 / 2}\right) .
$$

This also gives a good way for checking the computations by changing the value of $z$.

- For clarity we chose to split the sum $S$ at $x^{1 / 4}$, but in fact we only need to have $p \leq \frac{x}{p q}<p^{2}$. One can take advantage of this, but the asymptotic complexity remains the same.

- Precomputing the sieving by the first primes $2,3,5$ saves some more time. 


\section{Results}

The algorithm has been implemented in $C++$. All the computations were done using a HP 730 workstation (SPEC92INT=47.8). The 64-bit integers were emulated by the long long type of $\mathrm{GNU} \mathrm{C} / \mathrm{C}++$ Compiler.

For comparison we tried our program for some specific values on a DEC Alpha 3000 Model 600 at $175 \mathrm{Mhz}$ (which has 64-bit integers, SPEC92INT=114). The latter turned out to be more than three times faster. The difference could be greater because our program was optimized for a 32-bit computer, which is a drawback on a 64-bit computer.

We confirmed all the values already computed in [2]. Table 1 gives the new values compared with the corresponding values of

$$
\operatorname{Li}(x)=\int_{0}^{\infty} \frac{d t}{\log t}
$$

TABLE 1. Results and times of computation on HP-730

\begin{tabular}{|c|r|r|r|r|}
\hline$x$ & $\pi(x)$ & $\operatorname{Li}(x)-\pi(x)$ & $R(x)-\pi(x)$ & Time $(s)$ \\
\hline $1 \cdot 10^{15}$ & 29844570422669 & 1052619 & 73218 & 4179 \\
\hline $2 \cdot 10^{15}$ & 58478215681891 & 1317791 & -37631 & 6322 \\
\hline $3 \cdot 10^{15}$ & 86688602810119 & 1872580 & 233047 & 8110 \\
\hline $4 \cdot 10^{15}$ & 114630988904000 & 1364039 & -512689 & 9949 \\
\hline $5 \cdot 10^{15}$ & 142377417196364 & 2277608 & 193397 & 11572 \\
\hline $6 \cdot 10^{15}$ & 169969662554551 & 1886041 & -384694 & 12847 \\
\hline $7 \cdot 10^{15}$ & 197434994078331 & 2297328 & -144134 & 14115 \\
\hline $8 \cdot 10^{15}$ & 224792606318600 & 2727671 & 127929 & 15360 \\
\hline $9 \cdot 10^{15}$ & 252056733453928 & 1956031 & -791857 & 16608 \\
\hline $1 \cdot 10^{16}$ & 279238341033925 & 3214632 & 327052 & 17738 \\
\hline $2 \cdot 10^{16}$ & 547863431950008 & 3776488 & -225875 & 27690 \\
\hline $3 \cdot 10^{16}$ & 812760276789503 & 4651601 & -193899 & 35625 \\
\hline $4 \cdot 10^{16}$ & 1075292778753150 & 5538861 & -10980 & 42631 \\
\hline $5 \cdot 10^{16}$ & 1336094767763971 & 6977890 & 811655 & 48541 \\
\hline $6 \cdot 10^{16}$ & 1595534099589274 & 5572837 & -1147719 & 54266 \\
\hline $7 \cdot 10^{16}$ & 1853851099626620 & 8225687 & 997606 & 59615 \\
\hline $8 \cdot 10^{16}$ & 2111215026220444 & 6208817 & -1489898 & 64588 \\
\hline $9 \cdot 10^{16}$ & 2367751438410550 & 9034988 & 895676 & 69378 \\
\hline $10^{17}$ & 2623557157654233 & 7956589 & -598255 & 74369 \\
\hline $2 \cdot 10^{17}$ & 5153329362645908 & 10857072 & -1016134 & 115242 \\
\hline $3 \cdot 10^{17}$ & 7650011911220803 & 14592271 & 207129 & 148270 \\
\hline $4 \cdot 10^{17}$ & 10125681208311322 & 19808695 & 3323994 & 177024 \\
\hline $5 \cdot 10^{17}$ & 12585956566571620 & 19070319 & 747495 & 202791 \\
\hline $6 \cdot 10^{17}$ & 15034102021263820 & 20585416 & 609065 & 226471 \\
\hline $7 \cdot 10^{17}$ & 17472251499627256 & 18395468 & -3095204 & 253395 \\
\hline $8 \cdot 10^{17}$ & 19901908567967065 & 16763001 & -6132224 & 274919 \\
\hline $9 \cdot 10^{17}$ & 22324189231374849 & 26287786 & 2077405 & 293993 \\
\hline $1 \cdot 10^{18}$ & 24739954287740860 & 21949555 & -3501366 & 314754 \\
\hline
\end{tabular}


and

$$
R(x)=\sum_{n=1}^{\infty} \frac{\mu(n)}{n} \operatorname{Li}\left(x^{1 / n}\right) .
$$

\section{REFERENCES}

1. J. Bohman, On the number of primes less than a given limit, BIT 12 (1972), 576-578. MR 48 \#255

2. J. C. Lagarias, V. S. Miller, and A. M. Odlyzko, Computing $\pi(x)$ : The Meissel-Lehmer method, Math. Comp. 44 (1985), 537-560. MR 86h:11111

3. J. C. Lagarias and A. M. Odlyzko, Computing $\pi(x)$ : An analytic method, J. Algorithms 8 (1987), 173-191. MR 88k:11095

4. E. D. F. Meissel, Über die Bestimmung der Primzahlenmenge innerhalb gegebener Grenzen, Math. Ann. 2 (1870), 636-642.

5. _ Berechnung der Menge von Primzahlen, welche innerhalb der ersten hundert Millionen natürlicher Zahlen vorkommen, Math. Ann. 3 (1871), 523-525.

6. _ Über Primzahlenmengen, Math. Ann. 21 (1883), 304.

7. —_ Berechnung der Menge von Primzahlen, welche innerhalb der ersten Milliarde natürlicher Zahlen vorkommen, Math. Ann. 25 (1885), 289-292.

Département de Mathématiques, Université Lyon 1, 43 Blvd. Du 11 Novembre 1918, 69622 Villeurbanne Cedex, France

E-mail address: deleglis@lmdi.univ-lyon1.fr

E-mail address: rivat@caissa.univ-lyon1.fr 